\title{
HAT-P-28b AND HAT-P-29b: TWO SUB-JUPITER MASS TRANSITING PLANETS*†
}

\author{
L. A. Buchinave ${ }^{1,2}$, G. Á. Bakos ${ }^{1,11}$, J. D. Hartman ${ }^{1}$, G. Torres ${ }^{1}$, D. W. Latham $^{1}$, J. Andersen ${ }^{2,3}$, G. Kovács ${ }^{4}$, R. W. Noyes ${ }^{1}$,

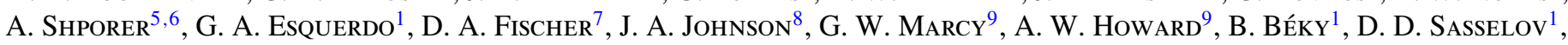

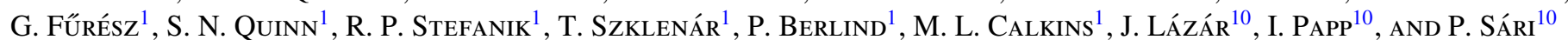 \\ ${ }^{1}$ Harvard-Smithsonian Center for Astrophysics, Cambridge, MA, USA \\ 2 Niels Bohr Institute, Copenhagen University, Denmark \\ ${ }^{3}$ Nordic Optical Telescope Scientific Association, La Palma, Canarias, Spain \\ ${ }^{4}$ Konkoly Observatory, Budapest, Hungary \\ ${ }^{5}$ Las Cumbres Observatory Global Telescope Network, Goleta, CA, USA \\ ${ }^{6}$ Department of Physics, University of California, Santa Barbara, CA, USA \\ ${ }^{7}$ Department of Astronomy, Yale University, New Haven, CT, USA \\ ${ }^{8}$ Department of Astrophysics, California Institute of Technology, Pasadena, CA, USA \\ ${ }^{9}$ Department of Astronomy, University of California, Berkeley, CA, USA \\ ${ }^{10}$ Hungarian Astronomical Association, Budapest, Hungary \\ Received 2011 January 17; accepted 2011 March 24; published 2011 May 16
}

\begin{abstract}
We present the discovery of two transiting exoplanets. HAT-P-28b orbits a $V=13.03 \mathrm{G} 3 \mathrm{dwarf}$ star with a period $P=3.2572$ days and has a mass of $0.63 \pm 0.04 M_{\mathrm{J}}$ and a radius of $1.21_{-0.08}^{+0.11} R_{\mathrm{J}}$ yielding a mean density of $0.44 \pm 0.09 \mathrm{~g} \mathrm{~cm}^{-3}$. HAT-P-29b orbits a $V=11.90 \mathrm{~F} 8$ dwarf star with a period $P=5.7232$ days and has a mass of $0.78_{-0.04}^{+0.08} M_{\mathrm{J}}$ and a radius of $1.11_{-0.08}^{+0.14} R_{\mathrm{J}}$ yielding a mean density of $0.71 \pm 0.18 \mathrm{~g} \mathrm{~cm}^{-3}$. We discuss the properties of these planets in the context of other known transiting planets.
\end{abstract}

Key words: planetary systems - stars: individual (HAT-P-28, GSC 2284-00503, HAT-P-29, GSC 3293-01539) techniques: photometric - techniques: spectroscopic

Online-only material: machine-readable tables

\section{INTRODUCTION}

When a planet transits its host star, we can detect not only the presence of the planet but also learn a great deal about its physical properties: we can infer the planet's mass and radius and thus its bulk density and composition, its temperature and atmospheric composition (Charbonneau et al. 2005), and the dynamics of the planetary system such as the projected spinorbit alignment (Winn et al. 2005). With over 100 transiting exoplanets (TEPs) discovered in wide-angle transit surveys and the wealth of transiting planet candidates from the Kepler mission (Borucki et al. 2011), statistical analyses are beginning to support constraints on the planet formation models (Triaud et al. 2010; Morton \& Johnson 2011).

Here we report the discovery of two new TEPs, HAT-P28b and HAT-P-29b, orbiting the stars GSC 2284-00503 and GSC 3293-01539, respectively. The Hungarian-made Automated Telescope Network (HATNet; Bakos et al. 2004) has been in operation since 2003 and has now covered approximately $14 \%$ of the sky, searching for TEPs around bright stars $(9.5 \lesssim r \lesssim 14.5)$. Although HAT-P-28b and HAT-P-29b are of the most common types of confirmed TEPs with respect to their mass and radii, they contribute to the characterization of the growing population of Jupiter-sized planets known to transit. The two discovered planets reported here orbit relatively faint

\footnotetext{
* Based in part on observations made with the Nordic Optical Telescope, operated on the island of La Palma jointly by Denmark, Finland, Iceland, Norway, and Sweden, in the Spanish Observatorio del Roque de los Muchachos of the Instituto de Astrofisica de Canarias.

$\dagger$ Based in part on observations obtained at the W. M. Keck Observatory, which is operated by the University of California and the California Institute of Technology. Keck time has been granted by NOAO (A201Hr) and NASA (N018Hr, N167Hr).

11 NSF Fellow.
}

stars compared to the current population of validated transiting planets, but the typical HATNet planets have relatively bright host stars compared to the wealth of planet candidates discovered by Kepler, and are hence more amenable to follow-up confirmation and characterization.

In Section 2, we briefly describe the initial photometric detection and subsequent spectroscopic and photometric followup observations used to confirm the planetary nature of HAT-P$28 \mathrm{~b}$ and HAT-P-29b. In Section 3, we describe the data analysis which we performed to determine the planetary and stellar parameters. Our findings are discussed in Section 4.

\section{OBSERVATIONS}

HAT-P-28b and HAT-P-29b were discovered by HATNet following an observational procedure which has been described in detail in several previous discovery papers (e.g., Bakos et al. 2010; Buchhave et al. 2010). Briefly, the procedure consists of the following steps.

1. Photometric observations with one or more of the HATNet telescopes to detect stars with periodic transit-like light curves.

2. High-resolution, low signal-to-noise ratio $(\mathrm{S} / \mathrm{N})$ "reconnaissance" spectroscopy observations to reject many false positives (such as M dwarf stars transiting G or F stars, or eclipsing binary stars blended with brighter giant stars).

3. Higher-precision photometric observations during transit to confirm the detection, show that it is consistent with a transiting planet, and use in determining the system parameters.

4. High-resolution, high-S/N "confirmation" spectroscopy to detect the orbital motion of the star due to the planet, characterize the host star, and rule out subtle blend scenarios. 
Table 1

Summary of Photometric Observations

\begin{tabular}{|c|c|c|c|c|}
\hline Instrument/Field ${ }^{\mathrm{a}}$ & Date(s) & Number of Images & Cadence (s) & Filter \\
\hline \multicolumn{5}{|c|}{ HAT-P-28b } \\
\hline HAT-7/163 & 2007 Sep-2008 Jan & 2329 & 330 & $R$ \\
\hline HAT-8/163 & 2007 Sep-2008 Jan & 1624 & 330 & $R$ \\
\hline HAT-6/164 & 2007 Sep-2008 Feb & 3685 & 330 & $R$ \\
\hline HAT-9/164 & 2007 Sep-2008 Feb & 2779 & 330 & $R$ \\
\hline KeplerCam $^{\mathrm{b}}$ & 2010 Sep 3 & 174 & 133 & Sloan $i$ \\
\hline KeplerCam ${ }^{\mathrm{b}}$ & 2010 Sep 6 & 129 & 163 & Sloan $i$ \\
\hline $\mathrm{FTN}^{\mathrm{c}}$ & 2010 Oct 2 & 476 & 35 & Sloan $i$ \\
\hline \multicolumn{5}{|c|}{ HAT-P-29b } \\
\hline HAT-5/089 & 2008 Oct-2009 Mar & 2779 & 330 & Sloan $r$ \\
\hline HAT-8/089 & 2008 Oct-2009 Mar & 674 & 330 & Sloan $r$ \\
\hline KeplerCam $^{\mathrm{b}}$ & 2011 Jan 1 & 199 & 73 & Sloan $i$ \\
\hline KeplerCam ${ }^{\mathrm{b}}$ & 2011 Jan 24 & 261 & 43 & Sloan $i$ \\
\hline
\end{tabular}

Notes.

${ }^{a}$ HAT-P-28 is in two HATNet fields, internally labeled as 163 and 164 . HAT-P-29 is in one field, labeled as 089.

b Observations from the KeplerCam CCD camera on the FLWO $1.2 \mathrm{~m}$ telescope.

${ }^{\mathrm{c}}$ Observations from the $2.0 \mathrm{~m}$ Faulkes Telescope North (FTN) at Haleakala Observatory in Hawaii.

Below we describe details of this procedure that are pertinent specifically to the discovery of HAT-P-28b and HAT-P-29b.

\subsection{Photometric Detection}

Table 1 summarizes the photometric observations of HAT-P$28 \mathrm{~b}$ and HAT-P-29b. Altogether 10,400 instrumental $R$-band 5.5 minute cadence photometric measurements of HAT-P-28 (GSC 2284-00503; 2MASS 00520018+3443422; $\alpha=00^{\mathrm{h}} 52^{\mathrm{m}} 00^{\mathrm{s}} .18$, $\delta=+34^{\circ} 43^{\prime} 42^{\prime \prime} .3$; J2000; $V=13.03$; Droege et al. 2006) were obtained with the HAT-6, HAT-7, HAT-8, and HAT9 telescopes. Three thousand four hundred and fifty-three Sloan $r$ measurements of HAT-P-29 (GSC 3293-01539; 2MASS $02123147+5146435 ; \alpha=02^{\mathrm{h}} 12^{\mathrm{m}} 31^{\mathrm{s}} .46, \delta=+51^{\circ} 46^{\prime} 43^{\prime \prime} .5$; $\mathrm{J} 2000 ; V=11.90$ ) were obtained with the HAT-5 and HAT8 telescopes.

These observations revealed box-like transit signals in the light curves of the stars with an apparent depth of $\sim 16.1 \mathrm{mmag}$ and $\sim 9.1 \mathrm{mmag}$, and a period of $P=3.2572$ days and $P=5.7232$ days, respectively (see Figures 1 and 2).

\subsection{Reconnaissance Spectroscopy}

High-resolution, low-S/N reconnaissance spectra were obtained for HAT-P-28 and HAT-P-29 using the HarvardSmithsonian Center for Astrophysics (CfA) Digital Speedometer (DS; Latham 1992) and the Tillinghast Reflector Échelle Spectrograph (TRES; Fúrész 2008) on the FLWO $1.5 \mathrm{~m}$ telescope as well as the FIber-fed Échelle Spectrograph (FIES) on the $2.5 \mathrm{~m}$ Nordic Optical Telescope (NOT) at La Palma, Spain (Djupvik \& Andersen 2010). The reconnaissance observations are listed in Table 2 and were used to estimate the effective temperature, surface gravity, and projected rotational velocity of the host stars as described by Torres et al. (2002). The procedures used for reduction and extraction of high-precision radial velocities of the FIES spectra are described in Buchhave et al. (2010).

The CfA DS reconnaissance observations revealed no detectable radial velocity (RV) variation at the $\sim 1 \mathrm{~km} \mathrm{~s}^{-1}$ precision of the observations. The TRES and FIES reconnaissance observations showed radial velocities with a low rms (HAT-P$28 \mathrm{~b}$ showed an rms of $12 \mathrm{~m} \mathrm{~s}^{-1}$ and the HAT-P-29 showed an rms of $82 \mathrm{~m} \mathrm{~s}^{-1}$ ), which both could be consistent with a plan-
Table 2

Summary of Reconnaissance Spectroscopy Observations

\begin{tabular}{cccccc}
\hline \hline Instrument & Date & $\begin{array}{c}T_{\text {eff } \star} \\
(\mathrm{K})\end{array}$ & $\begin{array}{c}\log g_{\star} \\
(\mathrm{cgs})\end{array}$ & $\begin{array}{c}v \sin i \\
\left(\mathrm{~km} \mathrm{~s}^{-1}\right)\end{array}$ & $\begin{array}{c}\gamma_{\mathrm{RV}}{ }^{\mathrm{a}} \\
\left(\mathrm{km} \mathrm{s}^{-1}\right)\end{array}$ \\
\hline \multicolumn{5}{c}{ HAT-P-28b } \\
\hline DS & 2009 Jan 13 & 5750 & 4.5 & 0 & +44.32 \\
DS & 2009 Jan 14 & 5750 & 4.5 & 0 & +42.34 \\
FIES & 2009 Jan 19 & 5750 & 4.5 & 2 & +43.85 \\
FIES & 2009 Jan 21 & 5750 & 4.5 & 2 & +43.87 \\
\hline \multicolumn{5}{c}{ HAT-P-29b } \\
\hline FIES & 2010 Aug 17 & 6000 & 4.0 & 6 & -21.67 \\
FIES & 2010 Aug 17 & 5750 & 4.0 & 6 & -21.76 \\
TRES & 2009 Jan 21 & 5750 & 4.5 & 6 & -21.59 \\
\hline
\end{tabular}

Note. ${ }^{\text {a }}$ The heliocentric RV of the target.

etary companion. Additionally, the spectra are consistent with the host stars being single, slowly rotating, dwarf stars.

\subsection{Photometric Follow-up Observations}

In-transit photometric observations of HAT-P-28 and HATP-29 were obtained with the KeplerCam CCD camera on the FLWO $1.2 \mathrm{~m}$ telescope and with the $2.0 \mathrm{~m}$ Faulkes Telescope North (FTN) at Haleakala Observatory in Hawaii. These observations are summarized in Table 1.

The images were reduced to light curves following the aperture photometry procedure described by Bakos et al. (2010). Figures 3 and 4 show the final trend-filtered light curves together with the best-fit transit model (see Section 3.2); the individual measurements are provided in Tables 3 and 4.

\subsection{High-resolution, High-S/N Spectroscopy}

We obtained high-resolution, high-S/N spectra of HAT-P-28 using NOT/FIES as well as HIRES (Vogt et al. 1994) mounted on the Keck I telescope on Mauna Kea, Hawaii. A total of 10 high-S/N FIES observations were obtained between 2009 October 3 and 18, and a total of eight HIRES observations, including six through an iodine gas absorption cell and two without the cell, were obtained between 2009 October 1 and 2010 February 23. For HAT-P-29, nine HIRES spectra were obtained between 2010 September 26 and December 14, one of which was without the iodine cell. 

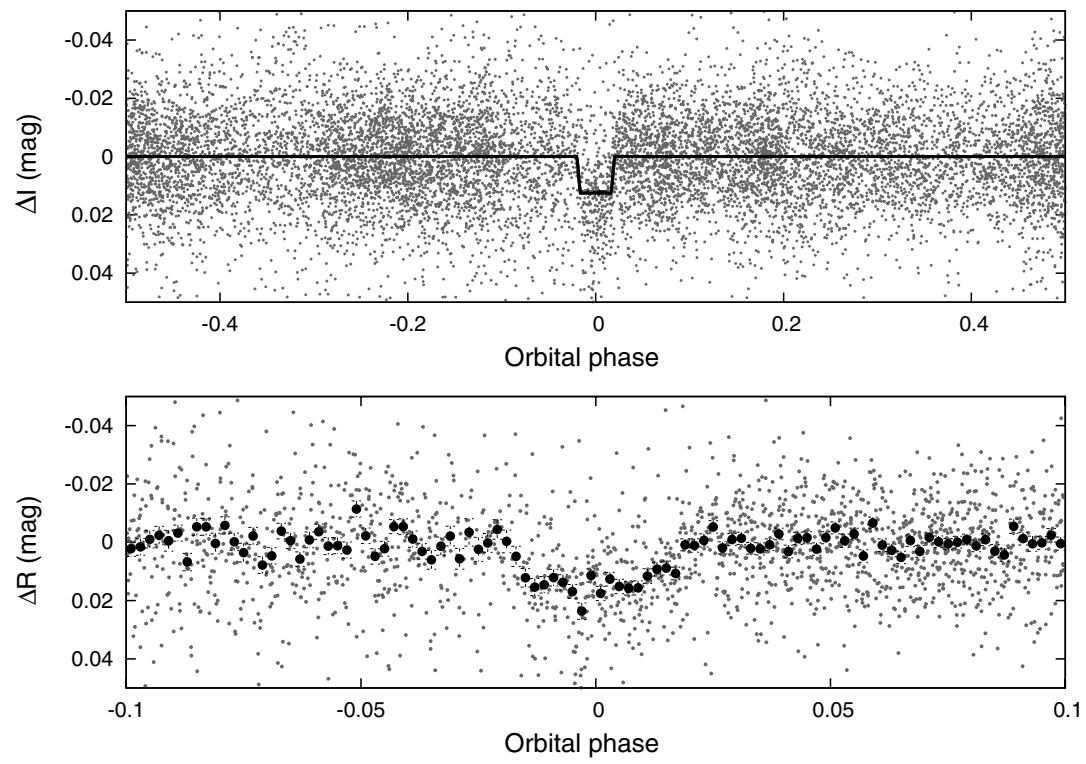

Figure 1. Unbinned light curve of HAT-P-28 including all 10,400 instrumental $R$-band 5.5 minute cadence measurements obtained with the HAT-6, HAT-7, HAT-8, and HAT-9 telescopes of HATNet (see Table 1), and folded with the period $P=3.257215 \pm 0.000007$ days resulting from the global fit described in Section 3 . The solid line shows a simplified transit model fit to the light curve (Section 3.2). The lower panel shows a zoomed-in view of the transit; the dark filled points show the light curve binned in phase using a bin size of 0.002 .
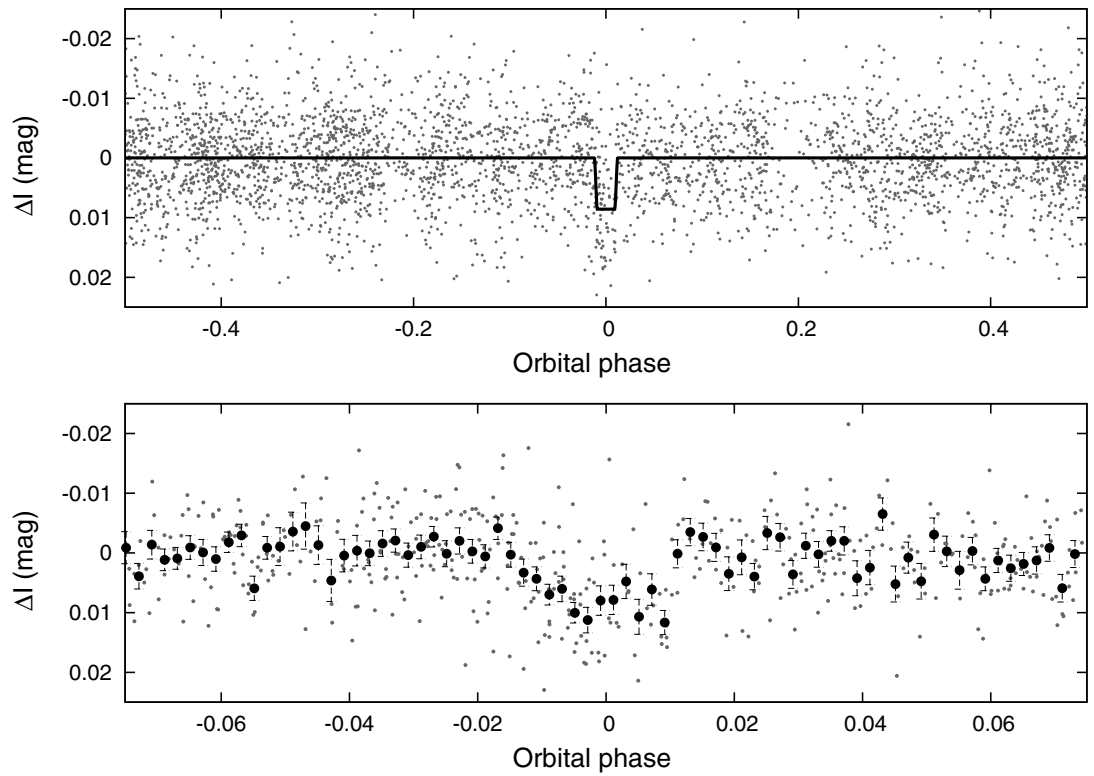

Figure 2. Unbinned light curve of HAT-P-29 including all 3453 instrumental Sloan $r 5.5$ minute cadence measurements obtained with the HAT-5 and HAT-8 telescopes and folded with the period $P=5.723186 \pm 0.000049$ days. See the caption of Figure 1 for details.

For the FIES observations, we used the medium-resolution fiber, giving a resolution of $\lambda / \Delta \lambda \approx 46,000$ and a wavelength coverage of $\sim 3600-7400 \AA$. The spectra were bracketed by ThAr calibration frames used to determine the fiducial wavelength calibration and were reduced to relative RVs in the solar system barycentric frame following a cross-correlation procedure described by Buchhave et al. (2010). For HIRES, we set the spectrometer slit to 0.86 , resulting in a resolving power of $\lambda / \Delta \lambda \approx 55,000$ with a wavelength coverage of $\sim 3800-8000 \AA$. We reduced these spectra to relative RVs in the solar system barycentric frame following a procedure based on that described by Butler et al. (1996). The RV measurements and their uncertainties are listed in Tables 5 and 6 . The period-folded data, along with our best fit described below in Section 3, are displayed in Figures 5 and 6.

Following Torres et al. (2007) and Queloz et al. (2001), we conducted a bisector span (BS) analysis as described in Section 5 of Bakos et al. (2007) of the Keck and FIES spectra to rule out the possibility that the objects are eclipsing binary star systems blended with light from a third star rather than a planet. As seen in Figures 5 and 6, the BS show no variation in phase with the orbital period and have a total scatter significantly less than the RV semiamplitude, which supports the interpretation that the velocity variations are due to planetary companions.

In the same figures, we also show the calibrated $S$ indices, which are a measure of the chromospheric activity of the stars 


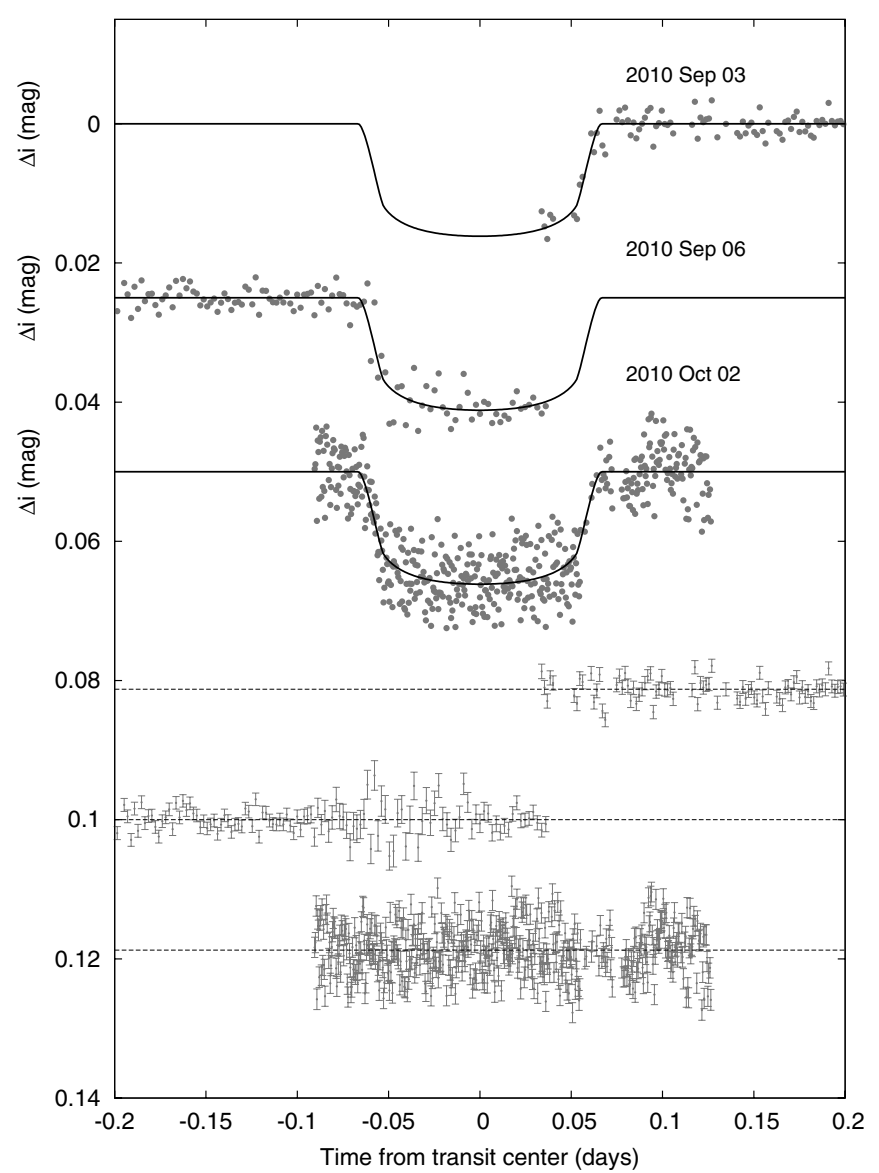

Figure 3. Unbinned instrumental Sloan $i$-band transit light curves of HAT-P-28, acquired with KeplerCam at the FLWO $1.2 \mathrm{~m}$ telescope on 2010 September 3 and 2010 September 6, and with the Faulkes Telescope North on 2010 October 2 , from top to bottom. The light curves have been cleaned of instrumental variations using the ELTG model (Section 3.2 and Bakos et al. 2010). Curves are displaced vertically for clarity. Our best fit from the global modeling described in Section 3.2 is shown by the solid lines. Residuals from the fits are displayed at the bottom, in the same order as the top curves. The error bars represent the photon and background shot noise, plus the readout noise.

Table 3

High-precision Differential Photometry of HAT-P-28

\begin{tabular}{ccccc}
\hline \hline $\begin{array}{c}\text { BJD } \\
(2,400,000+)\end{array}$ & Mag $^{\mathrm{a}}$ & $\sigma_{\mathrm{Mag}}$ & Mag(orig) & Filter \\
\hline 55443.68956 & 0.01257 & 0.00104 & 11.6842 & $i$ \\
55443.69111 & 0.01476 & 0.00102 & 11.6858 & $i$ \\
55443.69266 & 0.01656 & 0.00102 & 11.6878 & $i$ \\
55443.69421 & 0.01308 & 0.00102 & 11.6852 & $i$ \\
55443.69576 & 0.01364 & 0.00102 & 11.6776 & $i$ \\
55443.70735 & 0.01317 & 0.00100 & 11.6841 & $i$ \\
55443.70889 & 0.01369 & 0.00100 & 11.6852 & $i$ \\
55443.71044 & 0.00874 & 0.00099 & 11.6816 & $i$ \\
55443.71197 & 0.00762 & 0.00100 & 11.6786 & $i$ \\
55443.71663 & 0.00139 & 0.00100 & 11.6848 & $i$ \\
\hline
\end{tabular}

Notes.

a The out-of-transit level has been subtracted. These magnitudes have been subjected to the External Parameter Decorrelation (EPD) technique and Trend Filtering Algorithm (TFA) procedures (see Bakos et al. 2010), carried out simultaneously with the transit fit.

${ }^{\mathrm{b}}$ Raw magnitude values without application of the EPD and TFA procedures.

(This table is available in its entirety in a machine-readable form in the online journal. A portion is shown here for guidance regarding its form and content.)

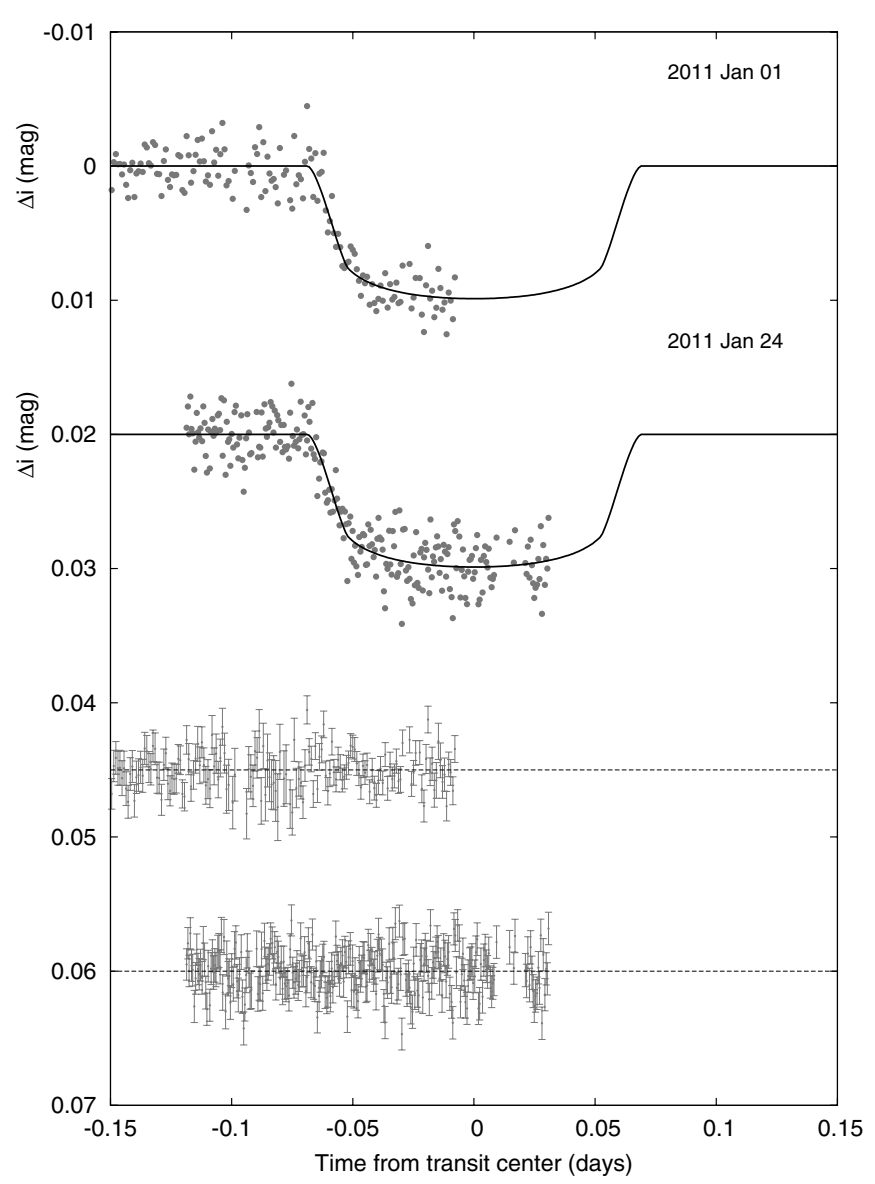

Figure 4. Unbinned instrumental Sloan $i$-band transit light curves of HAT-P-29, acquired with KeplerCam at the FLWO $1.2 \mathrm{~m}$ telescope on 2011 January 1 and January 24. See the caption in Figure 3 for details.

Table 4

High-precision Differential Photometry of HAT-P-29

\begin{tabular}{crccc}
\hline \hline $\begin{array}{c}\text { BJD } \\
(2,400,000+)\end{array}$ & \multicolumn{1}{c}{ Mag $^{\mathrm{a}}$} & $\sigma_{\text {Mag }}$ & Mag(orig) & Filter \\
\hline 55563.67299 & 0.00148 & 0.00114 & 10.8583 & $i$ \\
55563.67366 & 0.00031 & 0.00115 & 10.8581 & $i$ \\
55563.67444 & -0.00043 & 0.00099 & 10.8565 & $i$ \\
55563.67528 & -0.00158 & 0.00099 & 10.8556 & $i$ \\
55563.67614 & -0.00072 & 0.00097 & 10.8564 & $i$ \\
55563.67700 & 0.00009 & 0.00096 & 10.8567 & $i$ \\
55563.67784 & -0.00134 & 0.00094 & 10.8549 & $i$ \\
55563.67870 & 0.00242 & 0.00095 & 10.8600 & $i$ \\
55563.67955 & 0.00023 & 0.00094 & 10.8577 & $i$ \\
55563.68042 & 0.00100 & 0.00095 & 10.8581 & $i$
\end{tabular}

Notes.

a The out-of-transit level has been subtracted. These magnitudes have been subjected to the External Parameter Decorrelation (EPD) technique and Trend Filtering Algorithm (TFA) procedures (see Bakos et al. 2010), carried out simultaneously with the transit fit.

${ }^{\mathrm{b}}$ Raw magnitude values without application of the EPD and TFA procedures.

(This table is available in its entirety in a machine-readable form in the online journal. A portion is shown here for guidance regarding its form and content.)

derived from the flux in the cores of the $\mathrm{Ca}$ II $\mathrm{H}$ and $\mathrm{K}$ lines (Vaughan et al. 1978; Isaacson \& Fischer 2010). We also measured the $R_{\mathrm{HK}}^{\prime}$ indices for the systems to be $\log R_{\mathrm{HK}}^{\prime}=$ -4.984 and $\log R_{\mathrm{HK}}^{\prime}=-5.105$, respectively, as described by 

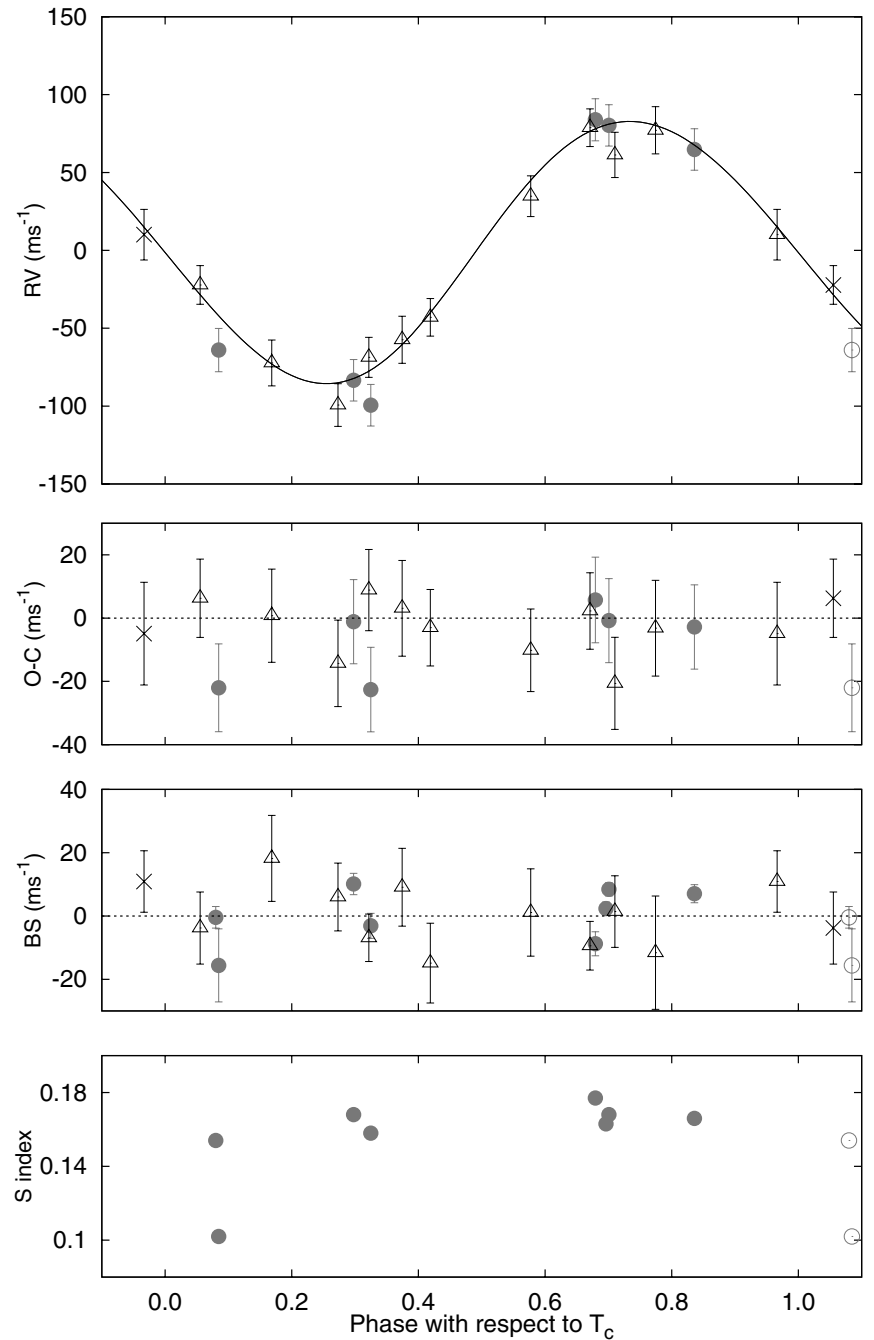

Figure 5. Top panel: high-precision relative RV measurements for HAT-P-28 from Keck/HIRES (filled circles) and NOT/FIES (open triangles) shown as a function of orbital phase, along with our best-fit model (see Table 8). Repeated points are shown as open circles and crosses for Keck/HIRES and NOT/FIES, respectively. Zero phase corresponds to the time of mid-transit. The centerof-mass velocity has been subtracted. Second panel: velocity $O-C$ residuals from the best fit. The error bars of the Keck/HIRES measurements include a component from astrophysical/instrumental jitter $\left(15.6 \mathrm{~m} \mathrm{~s}^{-1}\right.$; see Section 3.2); it was unnecessary to add jitter to the NOT/FIES measurements. The rms of the residuals is $16.1 \mathrm{~m} \mathrm{~s}^{-1}$ and $10.1 \mathrm{~m} \mathrm{~s}^{-1}$ for the Keck/HIRES and NOT/FIES observations, respectively. Third panel: bisector spans (BS), with the mean value subtracted. The measurement from the template spectrum is included. Bottom panel: relative chromospheric activity index $S$ measured from the Keck spectra. Note the different vertical scales of the panels.

Noyes et al. (1984). We do not detect any significant variation of the indices correlated with orbital phases.

\section{ANALYSIS}

The analysis of the HAT-P-28 and HAT-P-29 systems, including determinations of the properties of the host stars and planets, was carried out in a fashion similar to previous HATNet discoveries (e.g., Bakos et al. 2010). Below we briefly summarize the procedure and results.

\subsection{Properties of the Parent Star}

Stellar atmospheric parameters were measured using our template spectrum obtained with the Keck/HIRES instrument, and the analysis package known as Spectroscopy Made Easy (SME;
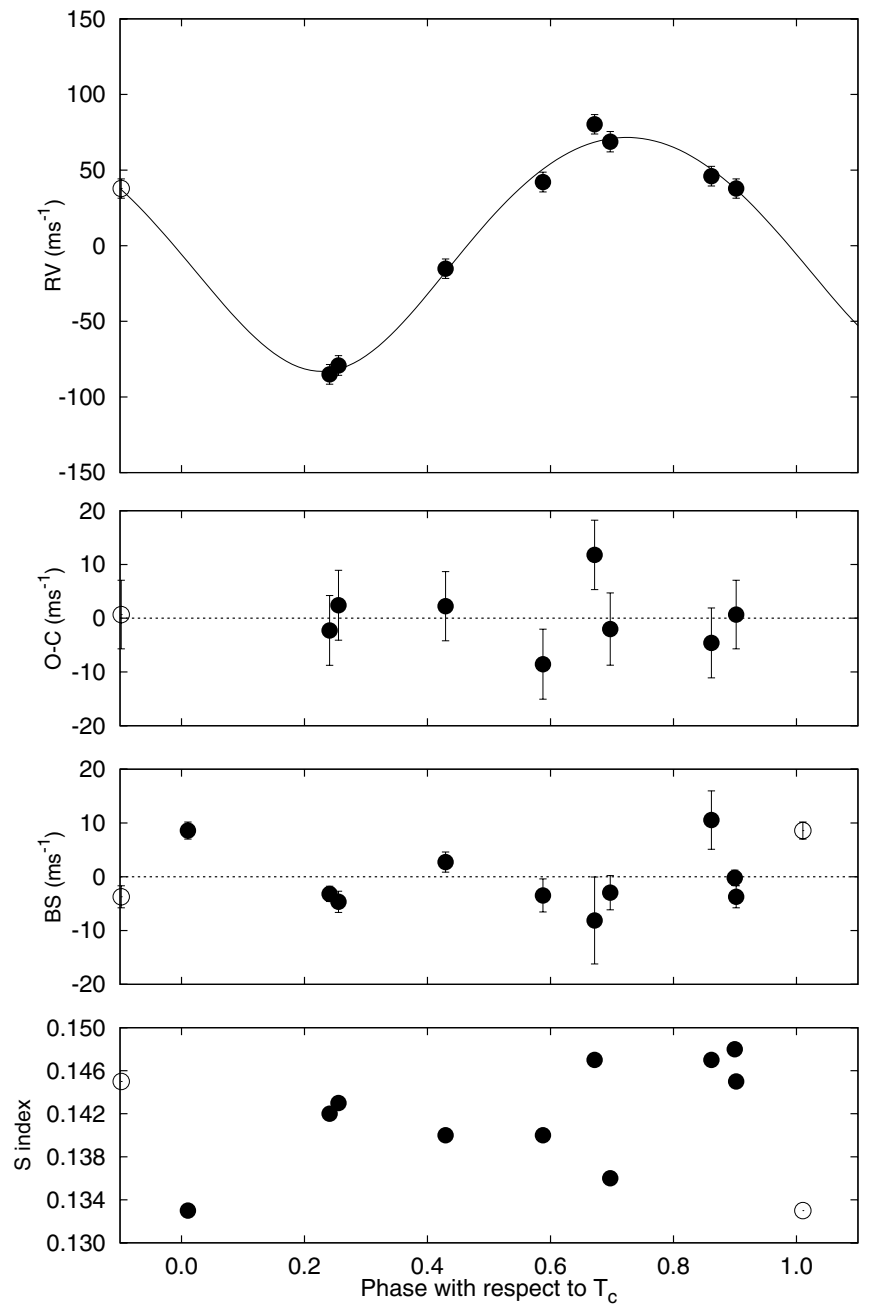

Figure 6. Top panel: high-precision relative RV measurements for HAT-P-29 from Keck/HIRES shown as a function of orbital phase, along with our best-fit model (see Table 8). Zero phase corresponds to the time of mid-transit. The center-of-mass velocity has been subtracted. Repeated points are shown as open circles. Second panel: velocity $O-C$ residuals from the best fit. The error bars of the Keck/HIRES measurements include a component from astrophysical/ instrumental jitter $\left(6.0 \mathrm{~m} \mathrm{~s}^{-1}\right.$; see Section 3.2). The rms of the residuals is $6.5 \mathrm{~m} \mathrm{~s}^{-1}$. Third panel: bisector spans (BS), with the mean value subtracted. Bottom panel: relative chromospheric activity index $S$ measured from the Keck spectra. Note the different vertical scales of the panels.

Valenti \& Piskunov 1996), along with the atomic-line database of Valenti \& Fischer (2005). SME yielded initial values and uncertainties (which, for the first two parameters, we have conservatively doubled to include our estimates of the systematic errors based on prior experience; see, e.g., Noyes et al. 2008). For HAT-P-28 we found the following parameters: effective temperature $T_{\text {eff } \star}=5680 \pm 90 \mathrm{~K}$, metallicity $[\mathrm{Fe} / \mathrm{H}]=+0.12 \pm$ 0.08 dex, projected rotational velocity $v \sin i=0.2_{-0.2}^{+0.5} \mathrm{~km} \mathrm{~s}^{-1}$, and stellar surface gravity $\log g_{\star}=4.42 \pm 0.06(\mathrm{cgs})$. For HAT-P-29 we found: effective temperature $T_{\text {eff } \star}=6087 \pm 88 \mathrm{~K}$, metallicity $[\mathrm{Fe} / \mathrm{H}]=0.21 \pm 0.08$ dex, projected rotational velocity $v \sin i=3.9 \pm 0.5 \mathrm{~km} \mathrm{~s}^{-1}$, and stellar surface gravity $\log g_{\star}=4.31 \pm 0.06(\mathrm{cgs})$.

Our initial values of $T_{\text {eff }}, \log g_{\star}$, and $[\mathrm{Fe} / \mathrm{H}]$ were used to determine the limb-darkening coefficients needed in the global modeling of the follow-up photometry. This modeling is briefly summarized in Section 3.2 with more details given by Bakos et al. (2010). Following Sozzetti et al. (2007) we used $a / R_{\star}$ (the normalized semimajor axis), $T_{\mathrm{eff} \star}$, and $[\mathrm{Fe} / \mathrm{H}]$ together with the 
Table 5

Relative Radial Velocities, Bisector Spans, and Activity Index Measurements of HAT-P-28

\begin{tabular}{lccccccc}
\hline \hline $\begin{array}{c}\text { BJD } \\
(2,400,000+)\end{array}$ & $\begin{array}{c}\mathrm{RV}^{\mathrm{a}} \\
\left(\mathrm{m} \mathrm{s}^{-1}\right)\end{array}$ & $\begin{array}{c}\sigma_{\mathrm{RV}}^{\mathrm{b}} \\
\left(\mathrm{m} \mathrm{s}^{-1}\right)\end{array}$ & $\begin{array}{c}\mathrm{BS} \\
\left(\mathrm{m} \mathrm{s}^{-1}\right)\end{array}$ & $\begin{array}{c}\sigma_{\mathrm{BS}} \\
\left(\mathrm{m} \mathrm{s}^{-1}\right)\end{array}$ & $S^{\mathrm{c}}$ & Phase & Instrument \\
\hline 55107.11869 & 83.86 & 3.76 & -8.78 & 3.78 & 0.177 & 0.680 & Keck \\
55108.71101 & -72.31 & 12.10 & 18.20 & 13.60 & $\ldots$ & 0.168 & FIES \\
55110.68494 & 77.09 & 12.60 & -11.60 & 17.90 & $\ldots$ & 0.774 & FIES \\
55111.59971 & -22.21 & 9.10 & -3.80 & 11.40 & $\ldots$ & 0.055 & FIES \\
55112.63861 & -57.41 & 12.60 & 9.10 & 12.30 & $\ldots$ & 0.374 & FIES \\
55113.60539 & 78.79 & 8.70 & -9.40 & 7.70 & $\ldots$ & 0.671 & FIES \\
55114.56809 & 10.09 & 13.90 & 10.90 & 9.70 & $\ldots$ & 0.967 & FIES \\
55115.56626 & -99.31 & 10.80 & 6.00 & 10.70 & $\ldots$ & 0.273 & FIES \\
55116.55720 & 34.79 & 10.00 & 1.10 & 13.80 & $\ldots$ & 0.577 & FIES \\
55122.55573 & -43.01 & 8.70 & -14.90 & 12.60 & $\ldots$ & 0.419 & FIES \\
55123.50498 & 61.29 & 11.90 & 1.40 & 11.30 & $\ldots$ & 0.710 & FIES \\
55125.49641 & -68.71 & 9.70 & -6.90 & 7.50 & $\ldots$ & 0.322 & FIES \\
55191.86077 & $\ldots$ & $\ldots$ & 2.33 & 2.14 & 0.163 & 0.696 & Keck \\
55191.87545 & 80.26 & 2.74 & 8.43 & 2.12 & 0.168 & 0.701 & Keck \\
55193.81948 & -83.44 & 2.85 & 10.12 & 3.37 & 0.168 & 0.298 & Keck \\
55198.83008 & 64.79 & 2.92 & 7.03 & 2.85 & 0.166 & 0.836 & Keck \\
55229.73714 & -99.43 & 3.16 & -3.11 & 3.96 & 0.158 & 0.325 & Keck \\
55251.74043 & $\ldots$ & $\ldots$ & -0.42 & 3.40 & 0.154 & 0.080 & Keck \\
55251.75531 & -64.03 & 4.85 & -15.61 & 11.55 & 0.102 & 0.085 & Keck \\
\hline
\end{tabular}

Notes. For the iodine-free template exposures there is no RV measurement, but the BS and $S$ index can still be determined. a The zero point of these velocities is arbitrary. An overall offset $\gamma_{\text {rel }}$ fitted separately to the FIES and Keck velocities in Section 3.2 has been subtracted.

${ }^{\mathrm{b}}$ Internal errors excluding the component of astrophysical/instrumental jitter considered in Section 3.2.

${ }^{c}$ Relative chromospheric activity index, calibrated to the scale of Vaughan et al. (1978).

Table 6

Relative Radial Velocities, Bisector Spans, and Activity Index Measurements of HAT-P-29

\begin{tabular}{lccccccc}
\hline \hline $\begin{array}{c}\text { BJD } \\
(2,400,000+)\end{array}$ & $\begin{array}{c}\mathrm{RV}^{\mathrm{a}} \\
\left(\mathrm{m} \mathrm{s}^{-1}\right)\end{array}$ & $\begin{array}{c}\sigma_{\mathrm{RV}}^{\mathrm{b}} \\
\left(\mathrm{m} \mathrm{s}^{-1}\right)\end{array}$ & $\begin{array}{c}\mathrm{BS} \\
\left(\mathrm{m} \mathrm{s}^{-1}\right)\end{array}$ & $\begin{array}{c}\sigma_{\mathrm{BS}} \\
\left(\mathrm{m} \mathrm{s}^{-1}\right)\end{array}$ & $S^{\mathrm{c}}$ & Phase & $\begin{array}{c}\text { Instrument } \\
55465.99028\end{array}$ \\
$\ldots$ & $\ldots$ & -0.20 & 1.44 & 0.148 & 0.900 & Keck \\
55466.00284 & 37.80 & 2.16 & -3.72 & 2.05 & 0.145 & 0.902 & Keck \\
55467.94293 & -85.06 & 2.47 & -3.19 & 1.42 & 0.142 & 0.241 & Keck \\
55469.02138 & -15.21 & 2.31 & 2.73 & 1.87 & 0.140 & 0.429 & Keck \\
55469.92851 & 42.10 & 2.53 & -3.47 & 3.07 & 0.140 & 0.588 & Keck \\
55490.91798 & -79.20 & 2.51 & -9.19 & 1.97 & 0.143 & 0.255 & Keck \\
55500.96329 & $\ldots$ & $\ldots$ & 3.48 & 1.14 & 0.133 & 0.010 & Keck \\
55521.91781 & 80.29 & 2.39 & -6.04 & 7.56 & 0.147 & 0.672 & Keck \\
55523.00463 & 45.97 & 2.49 & 10.14 & 5.01 & 0.147 & 0.862 & Keck \\
55544.95590 & 68.78 & 3.04 & -7.18 & 3.45 & 0.136 & 0.697 & Keck \\
\hline
\end{tabular}

Notes. For the iodine-free template exposures there is no RV measurement, but the BS and $S$ index can still be determined.

a The zero point of these velocities is arbitrary. An overall offset $\gamma_{\text {rel }}$ fitted separately to the FIES and Keck velocities in Section 3.2 has been subtracted.

b Internal errors excluding the component of astrophysical/instrumental jitter considered in Section 3.2.

${ }^{c}$ Relative chromospheric activity index, calibrated to the scale of Vaughan et al. (1978).

Yonsei-Yale (YY) stellar evolution models (Yi et al. 2001) to determine probability distributions of other stellar properties (such as $\log g_{\star}, R_{\star}$, and $M_{\star}$ ). Note that $a / R_{\star}$ is related to the mean stellar density and is determined from the analysis of the light curves and RV curves. This procedure has been described in further detail by Pál (2009b).

The inferred location of the stars in a diagram of $a / R_{\star}$ versus $T_{\text {eff }}$ together with Yi et al. (2001) isochrones is shown in Figures 7 and 8 . The SME results for the surface gravity yielded $\log g_{\star}=4.36 \pm 0.06$ for HAT-P-28 and $\log g_{\star}=4.34 \pm 0.06$ for HAT-P-29. Both values are consistent with our initial SME analysis and we therefore did not carry out a second iteration of SME and adopted these values for $\log g_{\star}$ and the values of $T_{\text {eff }},[\mathrm{Fe} / \mathrm{H}]$, and $v \sin i$ stated above as the final atmospheric properties of the star. The stellar parameters are listed in Table 7. We find that HAT-P-28 has a mass $M_{\star}=1.025 \pm 0.047 M_{\odot}$, radius $R_{\star}=1.103_{-0.069}^{+0.091} R_{\odot}$, and an age of $6.1_{-1.9}^{+2.6} \mathrm{Gyr}$ and HAT-P-29 has a mass $M_{\star}=1.207 \pm 0.046 M_{\odot}$, radius $R_{\star}=$ $1.224_{-0.075}^{+0.133} R_{\odot}$, and an age of $2.2 \pm 1.0 \mathrm{Gyr}$, according to these models.

As a check on the stellar evolution modeling we compare the observed photometric color indices of the stars to the values predicted by the modeling. We use the near-infrared magnitudes from the Two Micron All Sky Survey (2MASS) Catalogue (Skrutskie et al. 2006), which for HAT-P-28 are $J_{2 \mathrm{MASS}}=11.561 \pm 0.020, H_{2 \mathrm{MASS}}=11.206 \pm 0.023$, and $K_{2 \mathrm{MASS}}=11.104 \pm 0.018$ and for HAT-P-29 are $J_{2 \mathrm{MASS}}=$ $10.648 \pm 0.023, H_{2 \mathrm{MASS}}=10.395 \pm 0.023$, and $K_{2 \mathrm{MASS}}=$ 


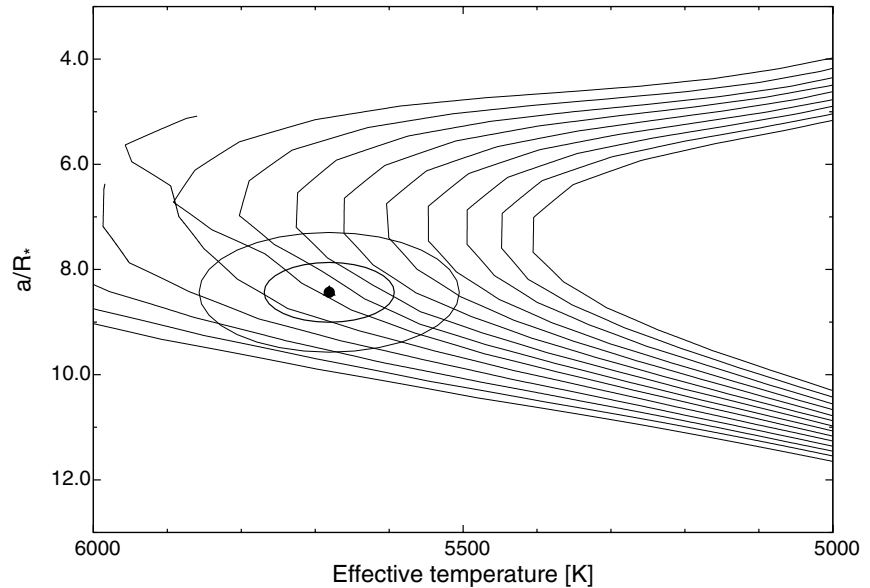

Figure 7. Model isochrones from Yi et al. (2001) for the measured metallicity of HAT-P-28, $[\mathrm{Fe} / \mathrm{H}]=+0.12$, and ages between 1 and $14 \mathrm{Gyr}$ in $1 \mathrm{Gyr}$ increments (left to right). The adopted values of $T_{\text {eff } \star}$ and $a / R_{\star}$ are shown together with their $1 \sigma$ and $2 \sigma$ confidence ellipsoids.

$10.297 \pm 0.020$. Converting these to the photometric system of the models (ESO) using the transformations by Carpenter (2001) we find a measured color index of $J-K=0.487 \pm 0.030$ and $J-K=0.375 \pm 0.033$, respectively. This is within $3 \sigma$ of the value predicted from the isochrones of $J-K=0.410 \pm 0.020$ and $J-K=0.340 \pm 0.020$, respectively.

From the observed and predicted $J-K$ we find $E(J-K)=$ $0.077 \pm 0.036$ and $E(J-K)=0.035 \pm 0.039$, respectively, and using Cardelli et al. (1989) we find $A(K)=0.052 \pm 0.024$ and $A(K)=0.024 \pm 0.026$, respectively, which gives a reddeningcorrected distance estimate, using the $K$ magnitude, of $395_{-26}^{+34} \mathrm{pc}$

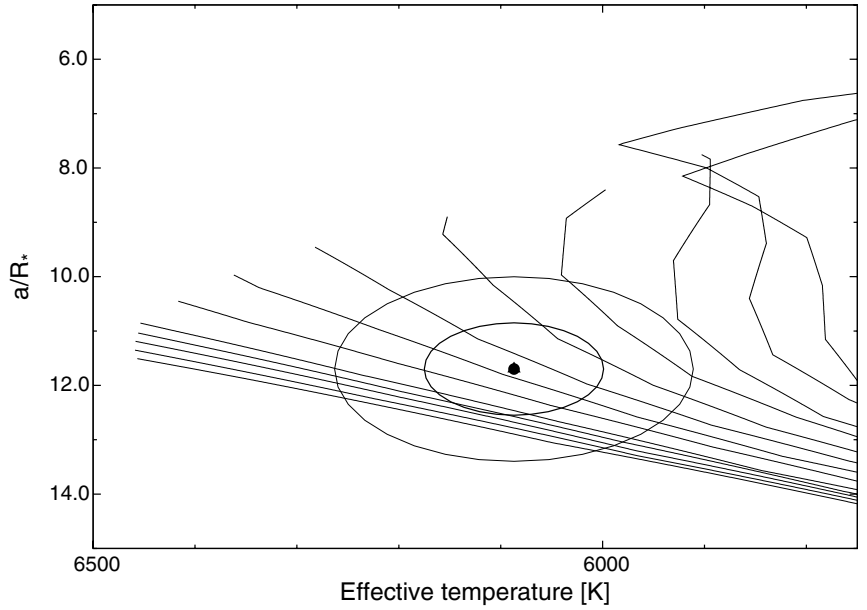

Figure 8. Model isochrones from Yi et al. (2001) for the measured metallicity of HAT-P-29, $[\mathrm{Fe} / \mathrm{H}]=0.21$, and ages between 1 and $13 \mathrm{Gyr}$ in $1 \mathrm{Gyr}$ increments (left to right). The adopted values of $T_{\text {eff } \star}$ and $a / R_{\star}$ are shown together with their $1 \sigma$ and $2 \sigma$ confidence ellipsoids.

for HAT-P-28 and $322_{-21}^{+35}$ pc for HAT-P-29. The uncertainty does not include difficult to quantify systematics in the model isochrones.

\subsection{Global Modeling of the Data}

We modeled the HATNet photometry, the follow-up photometry, and the high-precision RV measurements using the procedure described in detail by Bakos et al. (2010) and Pál (2009a). To summarize, we simultaneously fit these data using the Mandel \& Agol (2002) model for the follow-up light curves from KeplerCam and FTN, quadratic limb-darkening

Table 7

Stellar Parameters for HAT-P-28 and HAT-P-29

\begin{tabular}{|c|c|c|c|}
\hline Parameter & HAT-P-28 & HAT-P-29 & Source \\
\hline \multicolumn{4}{|l|}{ Spectroscopic properties } \\
\hline$T_{\mathrm{eff} \star}(\mathrm{K}) \ldots$ & $5680 \pm 90$ & $6087 \pm 88$ & SME $^{\mathrm{a}}$ \\
\hline$[\mathrm{Fe} / \mathrm{H}] \ldots$ & $+0.12 \pm 0.08$ & $0.21 \pm 0.08$ & SME \\
\hline$v \sin i\left(\mathrm{~km} \mathrm{~s}^{-1}\right) \ldots$ & $0.2_{-0.2}^{+0.5}$ & $3.9 \pm 0.5$ & SME \\
\hline$v_{\text {mac }}\left(\mathrm{km} \mathrm{s}^{-1}\right) \ldots$ & 3.88 & 4.50 & SME \\
\hline$v_{\text {mic }}\left(\mathrm{km} \mathrm{s}^{-1}\right) \ldots$ & 0.85 & 0.85 & SME \\
\hline $\begin{array}{l}\gamma_{\mathrm{RV}}\left(\mathrm{km} \mathrm{s}^{-1}\right) \ldots \\
\text { Photometric properties }\end{array}$ & $+43.884 \pm 0.068$ & $-21.670 \pm 0.08$ & FIES \\
\hline$V(\operatorname{mag}) \ldots$ & 13.03 & 11.90 & TASS \\
\hline$V-I_{C}(\mathrm{mag}) \ldots$ & $0.85 \pm 0.18$ & $0.85 \pm 0.18$ & TASS \\
\hline$J(\mathrm{mag}) \ldots$ & $11.561 \pm 0.020$ & $10.648 \pm 0.023$ & 2MASS \\
\hline$H(\operatorname{mag}) \ldots$ & $11.206 \pm 0.023$ & $10.395 \pm 0.023$ & 2MASS \\
\hline$K_{s}(\mathrm{mag}) \ldots$ & $11.104 \pm 0.018$ & $10.297 \pm 0.020$ & 2MASS \\
\hline \multicolumn{4}{|l|}{ Derived properties } \\
\hline $\begin{array}{l}M_{\star}\left(M_{\odot}\right) \ldots \\
R_{\star}\left(R_{\odot}\right) \ldots\end{array}$ & $\begin{array}{c}1.025 \pm 0.047 \\
1.103_{-0.069}^{+0.091}\end{array}$ & $\begin{array}{l}1.207 \pm 0.046 \\
1.224_{-0.075}^{+0.133}\end{array}$ & $\begin{array}{c}\mathrm{YY}+a / R_{\star}+\mathrm{SME}^{\mathrm{b}} \\
\mathrm{YY}+a / R_{\star}+\mathrm{SME}\end{array}$ \\
\hline $\begin{array}{l}\log g_{\star}(\operatorname{cgs}) \ldots \\
L_{\star}\left(L_{\odot}\right) \ldots\end{array}$ & $\begin{array}{l}4.36 \pm 0.06 \\
1.13_{-0.16}^{+0.23}\end{array}$ & $\begin{array}{l}4.34 \pm 0.06 \\
1.84_{-0.26}^{+0.47}\end{array}$ & $\begin{array}{l}\mathrm{YY}+a / R_{\star}+\mathrm{SME} \\
\mathrm{YY}+a / R_{\star}+\mathrm{SME}\end{array}$ \\
\hline$M_{V}(\mathrm{mag}) \ldots$ & $4.70 \pm 0.19$ & $4.11 \pm 0.21$ & $\mathrm{YY}+a / R_{\star}+\mathrm{SME}$ \\
\hline$M_{K}(\mathrm{mag}, \mathrm{ESO}) \ldots$ & $3.11 \pm 0.16$ & $2.77 \pm 0.19$ & $\mathrm{YY}+a / R_{\star}+\mathrm{SME}$ \\
\hline Age (Gyr)... & $6.1_{-1.9}^{+2.6}$ & $2.2 \pm 1.0$ & $\mathrm{YY}+a / R_{\star}+\mathrm{SME}$ \\
\hline Distance $(\mathrm{pc}) \ldots$ & $395_{-26}^{+34}$ & $322_{-21}^{+35}$ & $\mathrm{YY}+a / R_{\star}+\mathrm{SME}$ \\
\hline
\end{tabular}

Notes.

a SME: "Spectroscopy Made Easy" package for the analysis of high-resolution spectra (Valenti \& Piskunov 1996). These parameters rely primarily on SME, but have a small dependence also on the iterative analysis incorporating the isochrone search and global modeling of the data, as described in the text.

${ }^{\mathrm{b}} \mathrm{YY}+a / R_{\star}+\mathrm{SME}$ : based on the YY isochrones (Yi et al. 2001), $a / R_{\star}$ as a luminosity indicator and the SME results. 
Table 8

Orbital and Planetary Parameters

\begin{tabular}{|c|c|c|}
\hline Parameter & HAT-P-28b & HAT-P-29b \\
\hline \multicolumn{3}{|l|}{ Light curve parameters } \\
\hline$P($ days $) \ldots$ & $3.257215 \pm 0.000007$ & $5.723186 \pm 0.000049$ \\
\hline$T_{c}(\mathrm{BJD})^{\mathrm{a}} \ldots$ & $2455417.59832 \pm 0.00053$ & $2455197.57540 \pm 0.00181$ \\
\hline$T_{14}(\text { days) })^{\mathrm{a}} \ldots$ & $0.1341 \pm 0.0020$ & $0.1407 \pm 0.0074$ \\
\hline$T_{12}=T_{34}(\text { days })^{\mathrm{a}} \ldots$ & $0.0148 \pm 0.0015$ & $0.0177 \pm 0.0024$ \\
\hline$a / R_{\star} \ldots$ & $8.43 \pm 0.57$ & $11.70_{-0.97}^{+0.71}$ \\
\hline$\zeta / R_{\star} \ldots$ & $16.77 \pm 0.16$ & $16.22 \pm 0.93$ \\
\hline$R_{p} / R_{\star} \ldots$ & $0.1130 \pm 0.0024$ & $0.0927 \pm 0.0028$ \\
\hline$b^{2} \ldots$ & $0.089_{-0.052}^{+0.087}$ & $0.349_{-0.095}^{+0.079}$ \\
\hline$b \equiv a \cos i / R_{\star} \ldots$ & $0.299_{-0.123}^{+0.114}$ & $0.591_{-0.094}^{+0.062}$ \\
\hline$i(\operatorname{deg}) \ldots$ & $88.0 \pm 0.9$ & $87.1_{-0.7}^{+0.5}$ \\
\hline \multicolumn{3}{|l|}{ Limb-darkening coefficients ${ }^{\mathrm{b}}$} \\
\hline$a_{i}($ linear term $) \ldots$ & 0.2879 & 0.2273 \\
\hline$b_{i}$ (quadratic term)... & 0.3220 & 0.3581 \\
\hline \multicolumn{3}{|l|}{ RV parameters } \\
\hline$K\left(\mathrm{~m} \mathrm{~s}^{-1}\right) \ldots$ & $84.7 \pm 4.2$ & $78.3 \pm 5.9$ \\
\hline$e \cos \omega^{\mathrm{c}} \ldots$ & $-0.010 \pm 0.028$ & $-0.084_{-0.046}^{+0.026}$ \\
\hline$e \sin \omega^{\mathrm{c}} \ldots$ & $-0.022 \pm 0.053$ & $0.016 \pm 0.058$ \\
\hline e... & $0.051 \pm 0.033$ & $0.095 \pm 0.047$ \\
\hline$\omega(\operatorname{deg}) \ldots$ & $233 \pm 90$ & $169 \pm 30$ \\
\hline RV jitter HIRES $\left(\mathrm{m} \mathrm{s}^{-1}\right)^{\mathrm{d}} \ldots$ & 15.6 & 6.0 \\
\hline \multicolumn{3}{|l|}{ Secondary eclipse parameters } \\
\hline$T_{s}(\mathrm{BJD}) \ldots$ & $2455419.205 \pm 0.058$ & $2455200.132 \pm 0.138$ \\
\hline$T_{s, 14 \ldots}$ & $0.1289 \pm 0.0126$ & $0.1424 \pm 0.0107$ \\
\hline$T_{s, 12 \ldots}$ & $0.0142 \pm 0.0023$ & $0.0183 \pm 0.0074$ \\
\hline \multicolumn{3}{|l|}{ Planetary parameters } \\
\hline$M_{p}\left(M_{\mathrm{J}}\right) \ldots$ & $0.626 \pm 0.037$ & $0.778_{-0.040}^{+0.076}$ \\
\hline$R_{p}\left(R_{\mathrm{J}}\right) \ldots$ & $1.212_{-0.082}^{+0.113}$ & $1.107_{-0.082}^{+0.136}$ \\
\hline$C\left(M_{p}, R_{p}\right)^{\mathrm{e}} \ldots$ & 0.46 & 0.54 \\
\hline$\rho_{p}\left(\mathrm{~g} \mathrm{~cm}^{-3}\right) \ldots$ & $0.44 \pm 0.09$ & $0.71 \pm 0.18$ \\
\hline $\log g_{p}(\operatorname{cgs}) \ldots$ & $3.02 \pm 0.06$ & $3.20 \pm 0.07$ \\
\hline$a(\mathrm{AU}) \ldots$ & $0.0434 \pm 0.0007$ & $0.0667 \pm 0.0008$ \\
\hline$T_{\mathrm{eq}}(\mathrm{K}) \ldots$ & $1384 \pm 52$ & $1260_{-45}^{+64}$ \\
\hline$\Theta^{f^{f}} \ldots$ & $0.044 \pm 0.003$ & $0.077 \pm 0.007$ \\
\hline$F_{p e r}\left(10^{8} \mathrm{erg} \mathrm{s}^{-1} \mathrm{~cm}^{-2}\right)^{\mathrm{g}} \ldots$ & $9.16_{-0.92}^{+1.80}$ & $6.90_{-1.01}^{+3.61}$ \\
\hline$F_{a p}\left(10^{8} \mathrm{erg} \mathrm{s}^{-1} \mathrm{~cm}^{-2}\right)^{\mathrm{g}} \ldots$ & $7.58 \pm 1.34$ & $4.72_{-0.62}^{+0.81}$ \\
\hline$\langle F\rangle\left(10^{8} \mathrm{erg} \mathrm{s}^{-1} \mathrm{~cm}^{-2}\right)^{\mathrm{g}} \ldots$ & $8.28_{-1.06}^{+1.49}$ & $5.69_{-0.75}^{+1.36}$ \\
\hline
\end{tabular}

Notes.

${ }^{a} T_{c}$ : reference epoch of mid-transit that minimizes the correlation with the orbital period. BJD is calculated from UTC. $T_{14}$ : total transit duration, time between first to last contact; $T_{12}=T_{34}$ : ingress/egress time, time between first and second, or third and fourth contact.

b Values for a quadratic law, adopted from the tabulations by Claret (2004) according to the spectroscopic (SME) parameters listed in Table 7.

${ }^{c}$ Lagrangian orbital parameters derived from the global modeling, and primarily determined by the RV data.

${ }^{\mathrm{d}}$ This jitter was added to the Keck/HIRES measurements only. The NOT/FIES observations are consistent with no jitter.

${ }^{\mathrm{e}}$ Correlation coefficient between the planetary mass $M_{p}$ and radius $R_{p}$.

${ }^{\mathrm{f}}$ The Safronov number is given by $\Theta=\frac{1}{2}\left(V_{\text {esc }} / V_{\text {orb }}\right)^{2}=\left(a / R_{p}\right)\left(M_{p} / M_{\star}\right)$ (see Hansen \& Barman 2007).

$\mathrm{g}$ Incoming flux per unit surface area, averaged over the orbit.

coefficients interpolated from the tables by Claret (2004), a simplified no-limb-darkening transit model for the HATNet light curves, and a Keplerian orbit for the RVs.

The free parameters in the fit include the time of the first and last transit centers observed, the normalized planetary radius $p \equiv R_{p} / R_{\star}$, the square of the impact parameter $b^{2}$, the reciprocal of the half duration of the transit $\zeta / R_{\star}$, the RV semiamplitude $K$, Lagrangian elements $k \equiv e \cos \omega$ and $h \equiv e \sin \omega$, the HATNet blend factors (which account for possible dilution of the transit in the HATNet light curves from background stars due to the broad point spread function $\left(\sim 26^{\prime \prime}\right.$ FWHM $)$ and the application of trend filtering without signal reconstruction), the out-of-transit magnitudes for each HATNet field, the relative zero points $\gamma_{\text {rel,HIRES }}$ and $\gamma_{\text {rel,FIES }}$ of the HIRES/Keck and FIES/NOT RVs, and 22 instrumental parameters from the "ELTG" model for systematics.

We inflated the errors for the HIRES velocities by adding to quadrature a jitter of $15.6 \mathrm{~m} \mathrm{~s}^{-1}$ for HAT-P-28b and $6.0 \mathrm{~m} \mathrm{~s}^{-1}$ for HAT-P-29b to the formal errors to achieve a $\chi^{2} /$ dof $=1$. The internal uncertainty estimate of the FIES velocities, which is estimated by $\sigma=\operatorname{rms}(v) / \sqrt{N}$, where $v$ is the RVs of the individual orders and $N$ is the number of orders (see Buchhave et al. 2010), might tend to slightly overestimate the uncertainties of the individual measurements and thus we 


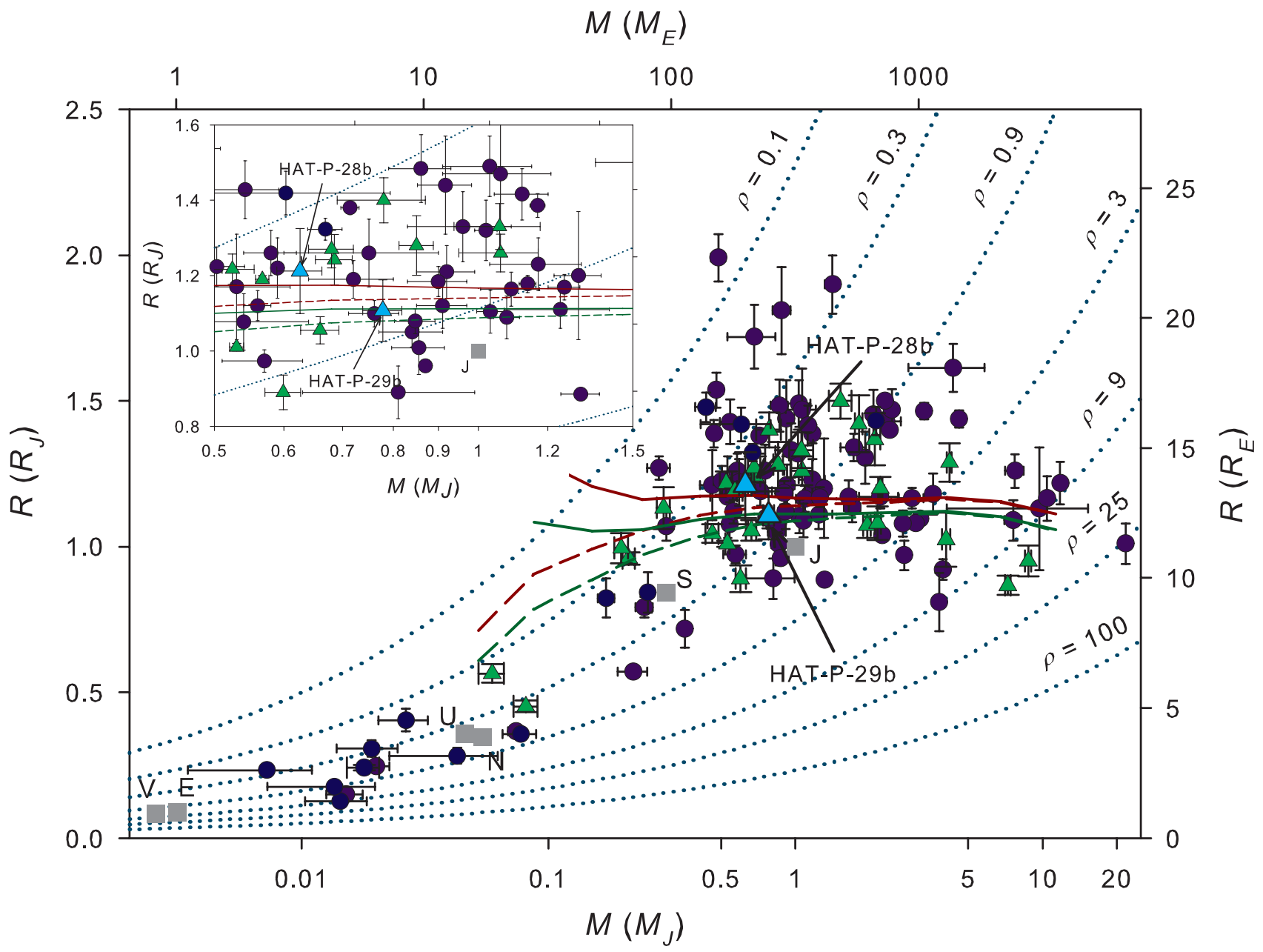

Figure 9. Mass-radius diagram of currently known TEPs. HATNet planets are shown as green triangles and TEPs from other surveys are shown as blue circles. HAT-P-28b and HAT-P-29b are shown as large light blue triangles. The solar system planets are shown as filled gray squares. The insert in the top left corner is a zoom of the region where HAT-P-28b and HAT-P-29b reside. Isodensity curves $\left(\right.$ in $\mathrm{g} \mathrm{cm}^{-3}$ ) are plotted as dotted lines. Overlaid are planetary $1.0 \mathrm{Gyr}$ (brown, the upper set of lines) and $4.5 \mathrm{Gyr}$ (green, the lower set of lines) isochrones from Fortney et al. (2007) for H/He-dominated planets with core masses of $M_{c}=0 M_{\oplus}$ (solid) and $M_{c}=10 M_{\oplus}$ (dashed), respectively, interpolated to the solar equivalent semimajor axis of HAT-P-28b. Given the estimated age of 6.1 $1_{-1.9}^{+2.6}$ Gyr, HAT-P-28b may be slightly larger than what is allowed by the theoretical models. HAT-P-29b is consistent with an H/He dominated planet with negligible core mass but also with a 1 Gyr model with a core mass of $M_{c}=10 M_{\oplus}$.

do not need to add jitter to the FIES velocities to achieve a $\chi^{2} /$ dof $=1$.

The derived planetary parameters can be found in Table 8 . We find:

1. HAT-P-28b: the planet has a mass of $M_{p}=0.626 \pm$ $0.037 M_{\mathrm{J}}$, a radius of $R_{p}=1.212_{-0.082}^{+0.113} R_{\mathrm{J}}$, and a mean density of $\rho_{p}=0.44 \pm 0.09 \mathrm{~g} \mathrm{~cm}^{-3}$.

2. HAT-P-29b: the planet has a mass of $M_{p}=0.778_{-0.040}^{+0.076} M_{\mathrm{J}}$, a radius of $R_{p}=1.107_{-0.082}^{+0.136} R_{\mathrm{J}}$, and a mean density of $\rho_{p}=0.71 \pm 0.18 \mathrm{~g} \mathrm{~cm}^{-3}$.

We find an eccentricity of $e=0.051 \pm 0.033$ and $e=$ $0.095 \pm 0.047$ for HAT-P-28b and HAT-P-29b, respectively, which are both consistent with a circular orbit.

\section{DISCUSSION}

We present the discovery of the transiting planets HAT-P$28 \mathrm{~b}$ and HAT-P-29b. Figure 9 shows the two planets on a mass-radius diagram comparing them to the other known TEPs. We discuss the properties of each planet below.

\subsection{HAT-P-28b}

HAT-P-28b has a period of $P=3.257215 \pm 0.000007$ days, a mass of $M_{p}=0.63 \pm 0.04 M_{\mathrm{J}}$, and a radius of $R_{p}=1.21_{-0.08}^{+0.11} R_{\mathrm{J}}$, leading to a mean density $\rho_{p}=0.44 \pm$ $0.09 \mathrm{~g} \mathrm{~cm}^{-3}$. We find an eccentricity of $e=0.051 \pm 0.033$, which is consistent with a circular orbit. Both Kepler-6b (Dunham et al. 2010) with $M_{p}=0.67 M_{\mathrm{J}}, R_{p}=1.32 R_{\mathrm{J}}$, $P=3.23$ days (Kipping \& Bakos 2010) and Kepler-8b (Jenkins et al. 2010) with $M_{p}=0.60 M_{\mathrm{J}}, R_{p}=1.42 R_{\mathrm{J}}, P=3.52$ days (Kipping \& Bakos 2010) are quite similar to HAT-P-28b in terms of their mass and periods, but have slightly larger radii. A significant number of other transiting planets have very comparable properties to HAT-P-28b. In fact, in histograms of mass and radius of the discovered transiting planets with a bin size of $0.5 M_{\mathrm{J}}$ and $0.25 R_{\mathrm{J}}$, respectively, HAT-P-28b resides in the most populated bins.

We have compared HAT-P-28b to the theoretical models from Fortney et al. (2007) by interpolating the models to the solar equivalent semimajor axis of $a=0.0434 \pm 0.0007 \mathrm{AU}$, the result of which can be seen overplotted in Figure 9. Given the estimated age of $6.1_{-1.9}^{+2.6} \mathrm{Gyr}$, we find that HAT-P-28b may be 
slightly larger than what is allowed by the theoretical models, but otherwise consistent with models of an $\mathrm{H} / \mathrm{He}$-dominated planet with a negligible core mass.

\subsection{HAT-P-29b}

HAT-P-29b has a period of $P=5.723186 \pm 0.000049$ days, a mass of $M_{p}=0.78_{-0.04}^{+0.08} M_{\mathrm{J}}$, and a radius of $R_{p}=1.11_{-0.08}^{+0.14} R_{\mathrm{J}}$, leading to a mean density $\rho_{p}=0.71 \pm 0.18 \mathrm{~g} \mathrm{~cm}^{-3}$, and has an eccentricity of $e=0.095 \pm 0.047$, which is consistent with a circular orbit. Again, HAT-P-29b is quite similar to a number of other transiting planets and is almost identical in mass and radius to TrES-1 $\left(M_{p}=0.75 M_{\mathrm{J}}, R_{p}=1.08 R_{\mathrm{J}}, P=3.03\right.$ days; Alonso et al. 2004), albeit with a slightly longer period.

When compared with the Fortney et al. (2007) planetary models, HAT-P-29b could be consistent with a $4.5 \mathrm{Gyr}$ model for an $\mathrm{H} / \mathrm{He}$-dominated planet with a negligible core mass but also with a 1 Gyr model with a core mass of $M_{c}=10 M_{\oplus}$.

HATNet operations have been funded by NASA grants NNG04GN74G, NNX08AF23G and SAO IR\&D grants. Work of G.Á.B. was supported by the Postdoctoral Fellowship of the NSF Astronomy and Astrophysics Program (AST-0702843 and AST-0702821, respectively). G.T. acknowledges partial support from NASA grant NNX09AF59G. We acknowledge partial support also from the Kepler mission under NASA Cooperative Agreement NCC2-1390 (PI: D.W.L.). G.K. thanks the Hungarian Scientific Research Foundation (OTKA) for support through grant K-81373. This research has made use of Keck telescope time granted through NOAO (program A201Hr) and NASA (N018Hr, N167Hr). This paper uses observations obtained with facilities of the Las Cumbres Observatory Global Telescope and the Nordic Optical Telescope, operated on the island of La Palma jointly by Denmark, Finland, Iceland, Norway, and Sweden, in the Spanish Observatorio del Roque de los Muchachos of the Instituto de Astrofisica de Canarias. We thank to Johan Fynbo for help on the distance estimate.

\section{REFERENCES}

Alonso, R., et al. 2004, ApJ, 613, L153

Bakos, G. Á., Noyes, R. W., Kovács, G., Stanek, K. Z., Sasselov, D. D., \& Domsa, I. 2004, PASP, 116, 266

Bakos, G. Á., et al. 2007, ApJ, 670, 826

Bakos, G. Á., et al. 2010, ApJ, 710, 1724

Borucki, W. J., et al. 2011, arXiv:1102.0541

Buchhave, L. A., et al. 2010, ApJ, 720, 1118

Butler, R. P., et al. 1996, PASP, 108, 500

Cardelli, J. A., Clayton, G. C., \& Mathis, J. S. 1989, ApJ, 345, 245

Carpenter, J. M. 2001, AJ, 121, 2851

Charbonneau, D., et al. 2005, ApJ, 626, 523

Claret, A. 2004, A\&A, 428, 1001

Djupvik, A. A., \& Andersen, J. 2010, in Highlights of Spanish Astrophysics V, ed. J. M. Diego, L. J. Goicoechea, J. I. González-Serrano, \& J. Gorgas (Berlin: Springer), 211

Droege, T. F., Richmond, M. W., \& Sallman, M. 2006, PASP, 118, 1666

Dunham, E. W., et al. 2010, ApJ, 713, L136

Fortney, J. J., Marley, M. S., \& Barnes, J. W. 2007, ApJ, 659, 1661

Fürész, G. 2008, PhD thesis,Univ. Szeged, Hungary

Hansen, B. M. S., \& Barman, T. 2007, ApJ, 671, 861

Isaacson, H., \& Fischer, D. 2010, ApJ, 725, 875

Jenkins, J. M., et al. 2010, ApJ, 724, 1108

Kipping, D. M., \& Bakos, G. Á. 2010, ApJ, 730, 50

Latham, D. W. 1992, in ASP Conf. Ser. 32, IAU Coll. 135, Complementary Approaches to Double and Multiple Star Research, ed. H. A. McAlister \& W. I. Hartkopf (San Francisco, CA: ASP), 110

Mandel, K., \& Agol, E. 2002, ApJ, 580, L171

Morton, T. D., \& Johnson, J. A. 2011, ApJ, 729, 138

Noyes, R. W., Hartmann, L. W., Baliunas, S. L., Duncan, D. K., \& Vaughan, A. H. 1984, ApJ, 279, 763

Noyes, R. W., et al. 2008, ApJ, 673, L79

Pál, A. 2009a, MNRAS, 396, 1737

Pál, A. 2009b, PhD thesis, Eötvös Loránd Univ.

Queloz, D., et al. 2001, A\&A, 379, 279

Skrutskie, M. F., et al. 2006, AJ, 131, 1163

Sozzetti, A., et al. 2007, ApJ, 664, 1190

Torres, G., Neuhauser, R., \& Guenther, E. W. 2002, AJ, 123, 1701

Torres, G., et al. 2007, ApJ, 666, 121

Triaud, A. H. M. J., et al. 2010, A\&A, 524, A25

Valenti, J. A., \& Fischer, D. A. 2005, ApJS, 159, 141

Valenti, J. A., \& Piskunov, N. 1996, A\&AS, 118, 595

Vaughan, A. H., Preston, G. W., \& Wilson, O. C. 1978, PASP, 90, 267

Vogt, S. S., et al. 1994, Proc. SPIE, 2198, 362

Yi, S. K., et al. 2001, ApJS, 136, 417

Winn, J. N., et al. 2005, ApJ, 631, 1215 